\title{
Impaired long-term outcomes of patients with schizophrenia spectrum disorder after coronary artery bypass surgery: nationwide case-control study
}

Mika Kallio, Jyrki Korkeila, Markus Malmberg, Jarmo Gunn, Päivi Rautava, Päivi Korhonen and Ville Kytö

\section{Background}

Patients with schizophrenia spectrum disorder have increased risk of coronary artery disease.

\section{Aims}

To investigate long-term outcomes of patients with schizophrenia spectrum disorder and coronary artery disease after coronary artery bypass grafting surgery (CABG).

\section{Method}

Data from patients with schizophrenia spectrum disorder ( $n=$ 126) were retrospectively compared with propensity-matched (1:20) control patients without schizophrenia spectrum disorder $(n=2520)$ in a multicentre study in Finland. All patients were treated with CABG. The median follow-up was 7.1 years. The primary outcome was all-cause mortality.

\section{Results}

Patients with diagnosed schizophrenia spectrum disorder had an elevated risk of 10-year mortality after CABG, compared with control patients (42.7 v. 30.3\%; hazard ratio 1.56; 95\% Cl 1.132.17; $P=0.008$ ). Schizophrenia spectrum diagnosis was associated with a higher risk of major adverse cardiovascular events during follow-up (49.9 v. 32.6\%, subdistribution hazard ratio 1.59 $95 \% \mathrm{Cl} 1.18-2.15 ; P=0.003)$. Myocardial infarction

(subdistribution hazard ratio 1.86; $P=0.003$ ) and cardiovascular mortality (subdistribution hazard ratio $1.65 ; P=0.017$ ) were more frequent in patients with versus those without schizophrenia spectrum disorder, but there was no difference for stroke. Psychiatric ward admission, antipsychotic medication, antidepressant use and benzodiazepine use before CABG were not associated with outcome differences. After CABG, patients with schizophrenia spectrum disorder received statin therapy less often and had lower doses; the use of other cardiovascular medications was similar between schizophrenia spectrum and control groups.

\section{Conclusions}

Patients with schizophrenia spectrum disorder have higher longterm risks of death and major adverse cardiovascular events after CABG. The results underline the vulnerability of these patients and highlight the importance of intensive secondary prevention and risk factor optimisation.

\section{Keywords}

Cardiovascular; coronary artery disease; myocardial infarction; severe mental disorder; schizophrenia.

\section{Copyright and usage}

(c) The Author(s), 2022. Published by Cambridge University Press on behalf of the Royal College of Psychiatrists. This is an Open Access article, distributed under the terms of the creative Commons Attribution-NonCommercial-ShareAlike licence (https://creativecommons.org/licenses/by-nc-sa/4.0/), which permits non-commercial re-use, distribution, and reproduction in any medium, provided the same Creative Commons licence is included and the original work is properly cited. The written permission of Cambridge University Press must be obtained for commercial re-use.
Patients with schizophrenia have a substantially higher overall mortality rate compared with the general population, ${ }^{1-4}$ and cardiovascular disease is the major cause of death. ${ }^{5}$ Coronary artery disease (CAD) is the largest contributor to the life expectancy gap between patients with schizophrenia and the general population. ${ }^{6-9}$ Previous studies have associated schizophrenia with adverse clinical outcomes and increased early mortality after myocardial infarction. ${ }^{10-12}$ Inequalities in treatment might contribute to mortality disparities because patients with severe mental illness, especially schizophrenia spectrum disorder (SCZ), are less likely to be treated with invasive coronary interventions. ${ }^{13-16}$ Recent findings suggest that patients with schizophrenia benefit from revascularisation as much as the general population does, despite a higher 1-year mortality rate after the procedure. ${ }^{17}$ No long-term studies are available about patients with schizophrenia who are treated with coronary artery bypass grafting surgery (CABG); however, $\mathrm{CABG}$ is the standard invasive treatment of choice in severe multivessel or left-main CAD. ${ }^{18}$ Our aim was to investigate the longterm outcomes of patients with chronic SCZ treated with CABG, using a large nationwide registry. We hypothesized that patients with schizophrenia or other chronic SCZ have poorer prognoses than patients who have CAD without SCZ.

\section{Method}

\section{Study design}

We studied the outcomes of patients with SCZ compared with patients without SCZ after CABG. Baseline differences between study groups were balanced by using propensity score matching. The primary outcome of interest was 10-year mortality. Secondary outcomes were 10-year major adverse cardiovascular event (MACE; including cardiovascular death, myocardial infarction and stroke) and use of postoperative medication. Patients with CAD treated with primary CABG between 1 January 2004 and 31 December $2014(n=35067)$ were retrospectively identified from The Care Register for Healthcare in Finland (CRHF). This nationwide, mandated-by-law registry includes data on all hospital and emergency department admissions and major surgical procedures in Finland. ${ }^{19}$ CABG was performed in eight hospitals (six public and two private) during the study period in Finland, and all were included in the study. Patients with prior cardiac surgery; concomitant surgery of heart valves; surgery of the aorta, ventricles or pulmonary vasculature defects; bypass surgery using gastroepiploic arterial or prosthetic grafts 
and those with unavailable follow-up data $(0.23 \%)$, were excluded from the study (Supplementary Fig. 1 available at https://doi.org/10. 1192/bjo.2022.10). Patients with SCZ were identified by following diagnostic codes from the International Classification of Diseasease version 10 (ICD-10) ${ }^{20}$ : F20 (schizophrenia), F21 (schizotypal disorder) and F25 (schizoaffective disorder). Other SCZ diagnoses were excluded to assure diagnostic stability. ${ }^{1}$ SCZs were identified in the $\mathrm{CRHF}$ and the national registry for drug reimbursement permissions held by the Social Insurance Institute.

\section{Definitions}

Comorbidities were identified in the CRHF, the national registry of drug reimbursement permissions and the nationwide Finnish Cancer Registry, using previously described ICD-10 codes and applicable drug purchase reimbursement codes. ${ }^{21}$ Myocardial infarction (ICD-10 code I21* or I22*) and stroke (ICD-10 codes $\left.\mathrm{I} 60^{*}-\mathrm{I} 64^{*}\right)$ occurring during follow-up were detected from the CRHF and death certificates. Cardiovascular death was defined with an ICD-10 code of $\mathrm{I}^{*}$ as the underlying cause of death. Postoperative prescription medications (drugs purchased within 90 days after discharge) were identified with applicable anatomical therapeutic chemical (ATC) codes (Supplementary Tables 1 and 2). In Finland, all psychiatric and cardiovascular disease medications are available only via prescription and are captured in the nationwide registry. Purchase of antipsychotics and benzodiazepines, as well as ward treatment in psychiatric hospitals during the year preceding CABG, were identified in a subgroup of patients with SCZ who underwent CABG between 2005 and 2017, using ATC codes and the CRHF. Regular medication use was defined as four or more drug purchases within a year. Sequential admissions after CABG were combined when studying the duration of admission. Prescription drug purchase data (including ATC codes and purchase dates) and drug purchase reimbursement permission data were obtained from a nationwide registry held by the Social Insurance Institution of Finland. Mortality data were obtained from a nationwide cause-of-death registry held by Statistics Finland. These registries are mandated by law and fully cover the Finnish population. Follow-up ended on 31 December 2018.

\section{Permissions and patient and public involvement statement}

The authors assert that all procedures contributing to this work comply with the ethical standards of the relevant national and institutional committees on human experimentation and with the Helsinki Declaration of 1975 , as revised in 2008. According to Finnish legislation, no ethical approval or informed consent is needed for register-based studies. The legal basis for processing of personal data was public interest and scientific research (EU General Data Protection Regulation 2016/679, Article 6(1)(e) and Article 9(2)(j); Data Protection Act, Sections 4 and 6). The study was approved by the National Institute for Health and Welfare of Finland (permission number THL/2245/5.05.00/2019), the National Social and Health Data Permit Authority (permission number THL/164/14.02.00/2021), and Statistics Finland (permission number TK-53-484-20).

\section{Statistical analysis}

A propensity score based on age, gender, atrial fibrillation, cerebrovascular disease, chronic pulmonary disease, diabetes, heart failure, hypertension, malignancy, myocardial infarction (new and prior), peripheral vascular disease, rheumatic disease, renal failure, type of bypass graft, off-pump CABG, number of grafted anastomoses, surgical centre and year of surgery (Table 1) was created with

Table 1 Baseline features of patients who underwent coronary artery bypass with and without schizophrenia spectrum disorder

\begin{tabular}{|c|c|c|c|c|c|c|c|c|}
\hline \multirow[b]{2}{*}{ Variable } & \multicolumn{4}{|c|}{ All patients } & \multicolumn{4}{|c|}{ Matched patients } \\
\hline & $\begin{array}{l}\text { Schizophrenia spectrum } \\
\qquad n=126\end{array}$ & $\begin{array}{c}\text { Control } \\
n=29094\end{array}$ & $P$-value & SMD & $\begin{array}{l}\text { Schizophrenia spectrum } \\
\qquad n=126\end{array}$ & $\begin{array}{l}\text { Control } \\
n=2520\end{array}$ & $P$-value & SMD \\
\hline Age, years (s.d.) & $64.0(8.7)$ & $66.8(9.1)$ & 0.001 & 0.318 & $64.0(8.7)$ & $64.0(9.1)$ & 0.758 & 0.001 \\
\hline Male gender & 93 (73.8\%) & $22800(78.4)$ & 0.215 & 0.107 & 93 (73.8\%) & 1867 (74.1\%) & 0.821 & 0.006 \\
\hline Atrial fibrillation & $13(10.3 \%)$ & 3259 (11.2\%) & 0.754 & 0.029 & $13(10.3 \%)$ & 242 (9.6\%) & 0.405 & 0.024 \\
\hline Cerebrovascular disease & $13(10.3 \%)$ & 2419 (8.3\%) & 0.417 & 0.069 & $13(10.3 \%)$ & $263(10.4 \%)$ & 0.885 & 0.004 \\
\hline Chronic pulmonary disease & $18(14.3 \%)$ & 3165 (10.9\%) & 0.221 & 0.103 & $18(14.3 \%)$ & $345(13.7 \%)$ & 0.532 & 0.017 \\
\hline Diabetes & 41 (32.5\%) & 7785 (26.8\%) & 0.144 & 0.127 & 41 (32.5\%) & 822 (32.6\%) & 0.950 & 0.002 \\
\hline Heart failure & $24(19.1 \%)$ & 4483 (15.4\%) & 0.259 & 0.097 & 24 (19.1\%) & 478 (19.0\%) & 0.942 & 0.002 \\
\hline Hypertension & $67(53.2 \%)$ & $16033(55.1 \%)$ & 0.663 & 0.039 & 67 (53.2\%) & 1348 (53.5\%) & 0.821 & 0.006 \\
\hline Malignancy & $8(6.4 \%)$ & 2627 (9.0\%) & 0.295 & 0.101 & $8(6.4 \%)$ & 171 (6.8\%) & 0.516 & 0.018 \\
\hline Peripheral vascular disease & 9 (7.1\%) & $2209(7.6 \%)$ & 0.849 & 0.017 & 9 (7.1\%) & $153(6.1 \%)$ & 0.128 & 0.043 \\
\hline Prior myocardial infarction & $34(27.0 \%)$ & 6097 (21.0\%) & 0.097 & 0.142 & 34 (27.0\%) & $693(27.5 \%)$ & 0.674 & 0.012 \\
\hline Rheumatic disease & $8(6.4 \%)$ & $1326(4.6 \%)$ & 0.336 & 0.079 & $8(6.4 \%)$ & 167 (6.6\%) & 0.678 & 0.011 \\
\hline Renal failure & $3(2.4 \%)$ & $626(2.2 \%)$ & 0.483 & 0.062 & $3(2.4 \%)$ & $70(2.8 \%)$ & 0.369 & 0.025 \\
\hline Myocardial infarction ${ }^{a}$ & 19 (15.1\%) & 2745 (9.4\%) & 0.031 & 0.173 & 19 (15.1\%) & 395 (15.7\%) & 0.535 & 0.017 \\
\hline Type of bypass graft & & & 0.939 & 0.028 & & & 0.124 & 0.028 \\
\hline Only arterial & 20 (15.9\%) & 4310 (14.8\%) & & & 20 (15.9\%) & 382 (15.2\%) & & \\
\hline Only venous & $8(6.4 \%)$ & 1796 (6.2\%) & & & $8(6.4 \%)$ & 159 (6.3\%) & & \\
\hline Arterial and venous & 98 (77.8\%) & 22988 (79.0\%) & & & 98 (77.8\%) & 1979 (78.5\%) & & \\
\hline Off-pump surgery & $6(4.8 \%)$ & 910 (3.1\%) & 0.294 & 0.084 & $6(4.8 \%)$ & 118 (4.7\%) & 0.678 & 0.004 \\
\hline Number of anastomoses & & & 0.471 & 0.081 & & & 0.646 & 0.027 \\
\hline 1 & $18(14.3 \%)$ & 2833 (9.7\%) & & & 18 (14.3\%) & 313 (12.4\%) & & \\
\hline 2 & $18(14.3 \%)$ & 3941 (13.6\%) & & & 18 (14.3\%) & 385 (15.3\%) & & \\
\hline 3 & $34(27.0 \%)$ & 9128 (31.4\%) & & & 34 (27.0\%) & 786 (31.2\%) & & \\
\hline 4 & $36(28.6 \%)$ & 8306 (28.6\%) & & & $36(28.6 \%)$ & 675 (26.8\%) & & \\
\hline$\geq 5$ & 20 (15.9\%) & $4886(16.8 \%)$ & & & 20 (15.9\%) & $361(14.3 \%)$ & & \\
\hline Surgical centre $(n=8)$ & & & 0.002 & 0.423 & & & 0.349 & 0.025 \\
\hline Year of surgery & & & 0.007 & 0.250 & & & 0.348 & 0.035 \\
\hline
\end{tabular}




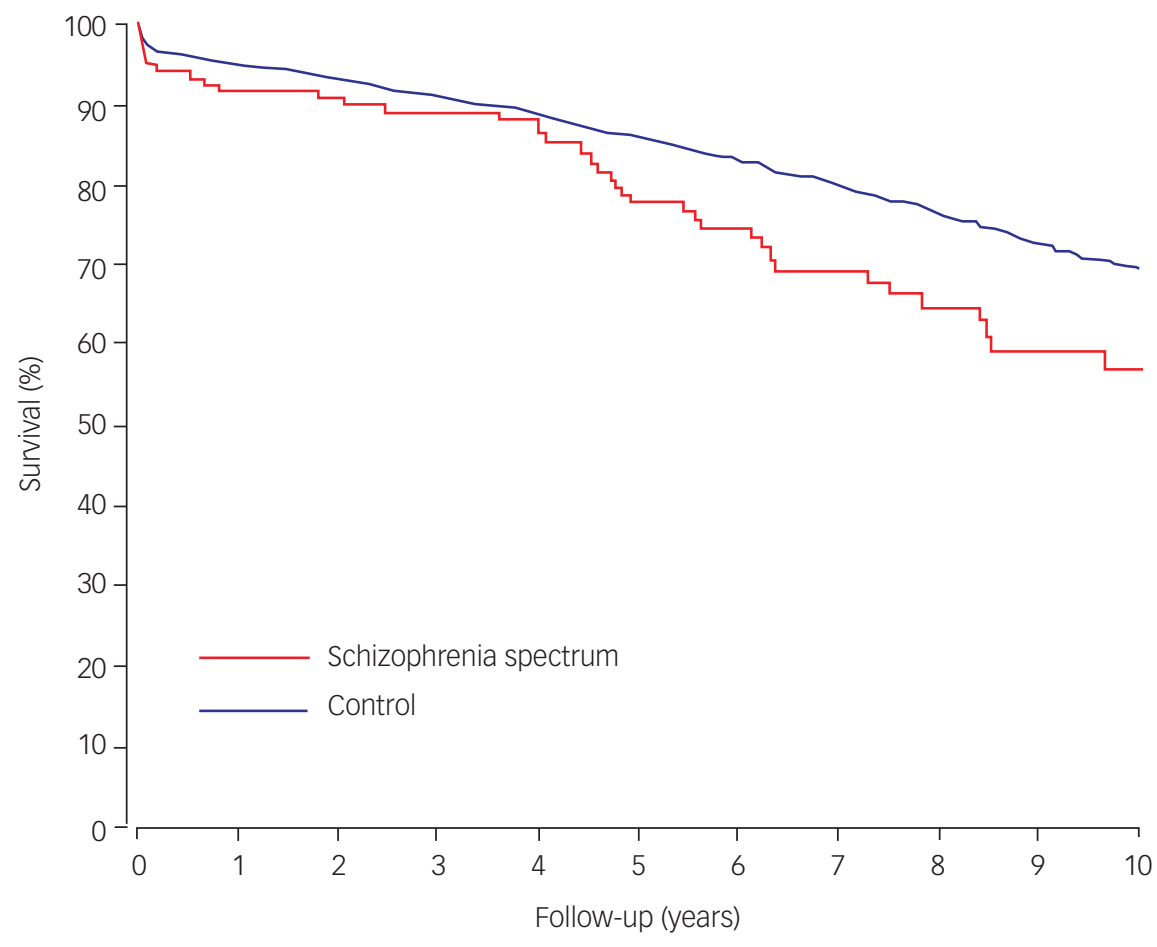

Fig. 1 Survival of patients with schizophrenia spectrum disorder and of matched controls patients after coronary artery bypass surgery.

logistic regression. Patients with nonoverlapping propensity scores were excluded ( $n=321$ patients without SCZ). A trimmed propensity score was used for local, optimal 1:20 calibre matching without replacement, using a 0.05 -caliper width of the logit of the s.d. ${ }^{22}$ Differences between nonmatched groups were studied with the $t$-test and the $\chi^{2}$-test, and differences betweenmatched groups were studied with the paired $t$-test, Wilcoxon signed-rank test and McNemar's test. Effect sizes in baseline characteristics between the study groups were evaluated by standardised difference scores. Use of prescription medications after CABG was studied with logistic regression. Time-dependent outcomes were studied with the Kaplan-Meier method and Cox regression (primary outcome), and with the Fine-Gray method to account for the competitive risk of death (secondary outcomes). Matched regression models were used in the analysis of the matched cohort. Multivariable regressions were used in the analysis of the subgroup of patients with SCZ who underwent operations between 2005 and $2017(n=118)$. Schoenfeld residuals were examined for confirmation of proportional hazard assumptions. The median follow-up time of matched patients was 7.1 years (interquartile range (IQR) 4.0-10.0 years), and there was no difference between the study groups $(P=0.567)$. Results are given as the mean, median, percentage, standardised mean difference, hazard ratio or odds ratio with $95 \%$ confidence intervals. Statistical significance was inferred by a $P$-value $<0.05$. Analyses were performed with SAS version 9.4 for Windows (SAS Institute Inc., Cary, North Carolina, USA).

\section{Results}

Of all included patients with CAD and treated with CABG ( $n=29220$; median age 68 years; $78.4 \%$ men), $0.4 \%(n=126)$ had SCZ. Patients with SCZ were younger and underwent operation because of myocardial infarction more often compared with patients in the control group (Table 1). Neither the type of bypass graft nor the number of grafted coronary anastomoses differed significantly between study groups. Propensity matching (1:20) identified 126 patients with SCZ and 2520 patients in the control group with balanced features (Table 1). The median duration of hospital admission after CABG was 14 days (IQR 10-25 days) in those with SCZ and 10 days (IQR 8-14 days) in matched controls $(P<0.001)$.

\section{Mortality}

During the 10-year follow-up of the matched cohort, 572 deaths occurred (37 in the SCZ group and 535 in matched controls). In-hospital mortality was $4.8 \%$ in the SCZ group and $2.9 \%$ in the control group $(P=0.364)$, and 30 -day mortality rates were $4.8 \%$ and $2.4 \%$, respectively $(P=0.095)$. Patients with and without SCZ had comparable all-cause mortality up to 4 years after CABG, but mortality was significantly higher in the SCZ group in the longterm follow-up (Fig. 1). Ten-year all-cause mortality was $42.7 \%$ in patients with SCZ and $30.3 \%$ in the control group after CABG (hazard ratio 1.56 ; $95 \%$ CI 1.13-2.17; $P=0.008$ ).

\section{MACE}

MACE occurred in 670 patients ( $n=46$ in the SCZ group) during the follow-up. The 30-day non-perioperative MACE incidence was $12.9 \%$ in patients with SCZ and $5.0 \%$ in the control group $(P=0.0001)$. The difference between the study groups persisted during follow-up (Fig. 2). The cumulative incidence of MACE was $49.9 \%$ in the SCZ group and $32.6 \%$ in the control group at the 10-year follow-up (subdistribution hazard ratio 1.59; $95 \%$ CI $1.18-2.15 ; P=0.003)$.

The cumulative incidence of myocardial infarction during the 10 -year follow-up was $28.8 \%$ in patients with SCZ compared with $15.3 \%$ in patients in the control group (subdistribution hazard ratio 1.86; 95\% CI 1.25-2.78; $P=0.003$; Supplementary Fig. 2). The 10 -year cumulative stroke incidence was $15.5 \%$ in the SCZ 


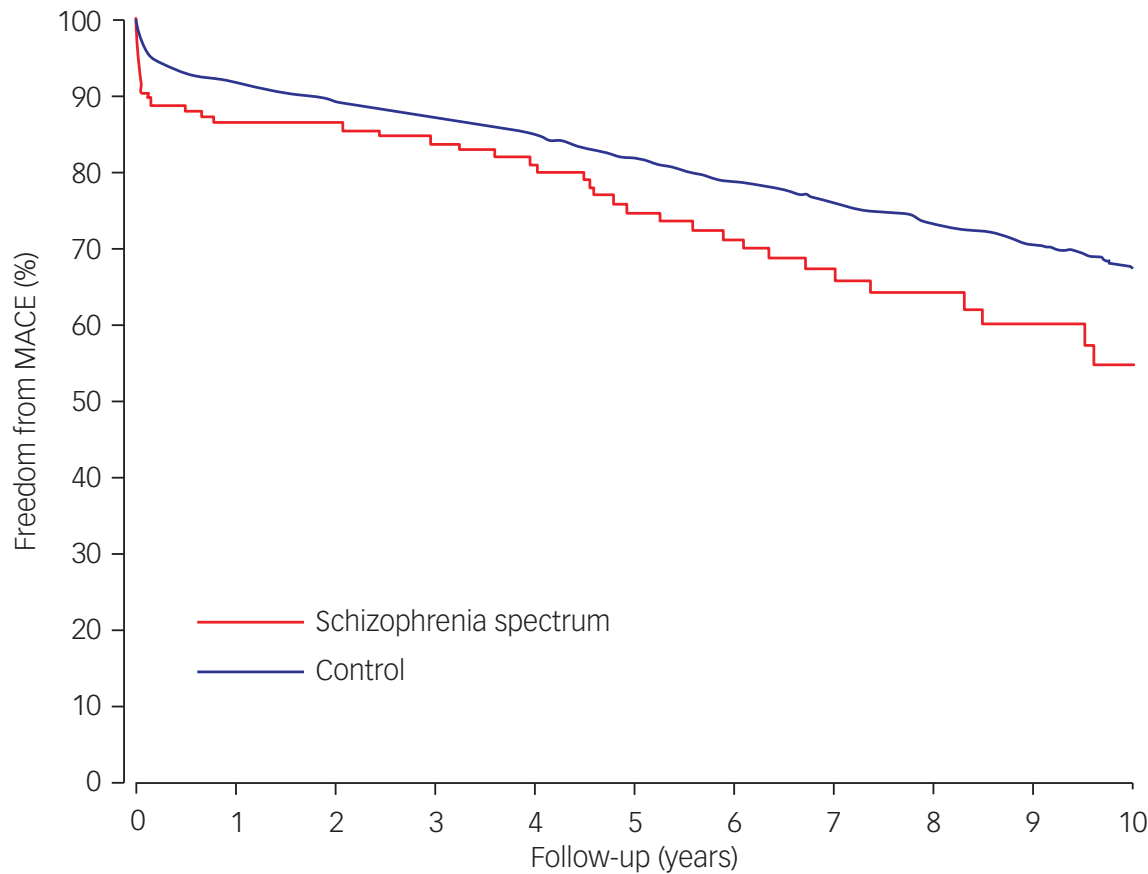

Fig. 2 Freedom from major adverse cardiovascular events (MACE) in patients with schizophrenia spectrum disorder and in matched control patients after coronary artery bypass grafting.

group and $14.1 \%$ in the control group (subdistribution hazard ratio $0.91 ; 95 \%$ CI $0.50-1.66 ; \quad P=0.765$; Supplementary Fig. 2). Cardiovascular mortality during follow-up was $26.7 \%$ in the SCZ group compared with $17.4 \%$ in the control group (subdistribution hazard ratio $1.65 ; 95 \%$ CI $1.10-2.48 ; P=0.017$; Supplementary Fig. 2).

\section{Postoperative medication}

Prescription medications after CABG are presented in Table 2. Statins were used less frequently by patients with SCZ than by controls $(80.8 v .87 .8 \% ; P=0.022)$. When statin therapy was used, high-intensity doses were less common in the SCZ compared with the non-SCZ group (12.4 v. 22.0\%; $P=0.023$ ). No significant differences were observed between patients with SCZ and matched controls in the use of antithrombotics, antidiabetics, antiarrhythmics, angiotensin pathway-targeting drugs, beta-blockers, calcium channel blockers, digitalis, diuretics or nitrates. Benzodiazepines, antidepressants and antipsychotics were used more frequently by patients with SCZ than those without SCZ after CABG (Table 2).

\section{Psychiatric hospital admissions and medications}

Nine (7.6\%) out of 118 patients with SCZ were treated in a psychiatric hospital ward in the year preceding CABG. The median length of hospital stay was 15 days (IQR 13-47 days). In the year preceding

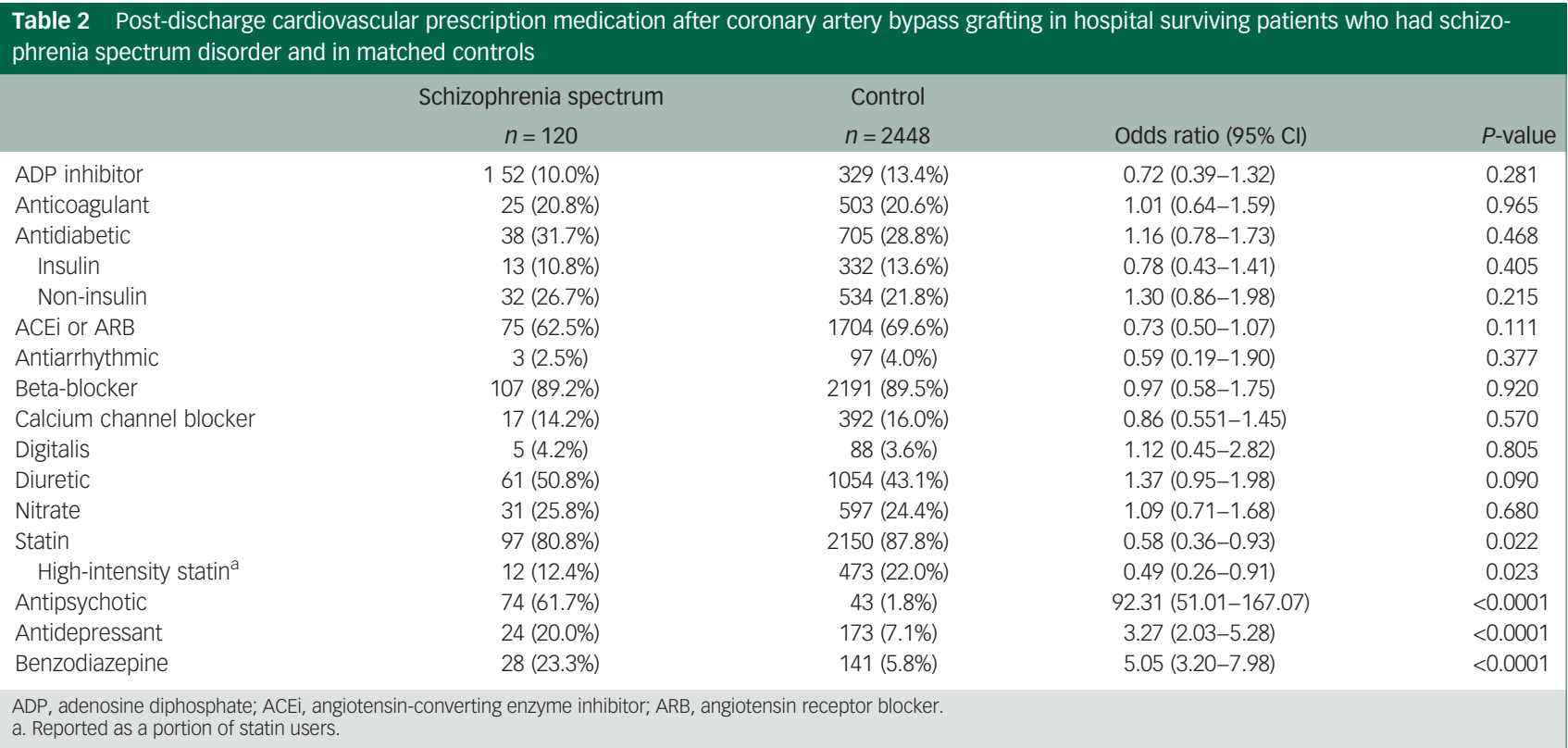


CABG, $75.8 \%$ of patients with SCZ used antipsychotic prescription medication, $28.0 \%$ used antidepressants and $22.0 \%$ used benzodiazepines. These medications were used regularly by $58.6 \%, 13.6 \%$ and $19.5 \%$ of patients with SCZ, respectively. Psychiatric ward admission or use of antipsychotics, antidepressants or benzodiazepines before CABG was not associated with long-term mortality or MACE (Supplementary Table 3).

\section{Discussion}

This multicentre, nationwide, retrospective case-control study showed that patients with SCZ had increased long-term all-cause mortality after CABG. MACE also occurred significantly more often in the SCZ group throughout the follow-up period, and appeared driven by increased cardiovascular mortality and myocardial infarction, but not by increased strokes. These results call attention to the need for aggressive secondary prevention in patients with SCZ and severe CAD.

Schizophrenia is a global health burden, resulting in up to 3.5fold the standardised mortality ${ }^{23}$ and a reduction in life expectancy by $15-25$ years compared with the general population., ${ }^{6,24}$ Cardiovascular mortality in schizophrenia is estimated to range from 40 to $50 \%$, and therefore is a major contributor to shortened life expectancy. ${ }^{25}$ In a previous register-based study, Attar et $\mathrm{al}^{26}$ examined patients with schizophrenia after acute coronary syndrome and found that they had a higher overall mortality (hazard ratio 2.54), greater risk of MACE (hazard ratio 1.62) and greater risk of stroke (hazard ratio 1.51) after acute coronary syndrome compared with patients without schizophrenia. Patients with SCZ seem less likely than the general population to receive revascularisation therapy after myocardial infarction, ${ }^{16}$ although revascularisation seems to benefit those with SCZ as much as it benefits the general population after myocardial infarction, at least during a 1year follow-up period. ${ }^{17}$ Studies investigating the long-term survival of patients with SCZ after revascularisation therapy are scarce; to our knowledge, this is the first study to explore outcomes after CABG.

We found an increased all-cause mortality in patients with SCZ during the 10-year follow-up after CABG, but, notably, this difference was present only beyond 4 years after CABG. In addition, our results show that patients with SCZ were approximately 1.5 times more likely to suffer from MACE during the long-term follow-up after CABG. The cumulative incidence of myocardial infarction during the 10-year follow-up was almost double in the SCZ group compared with the control group. Patients with SCZ also had a $40 \%$ longer duration of hospital admission than those in the control group. No previous studies about the long-term outcomes of patients with SCZ after CABG are available for a direct comparison with our results. In a recent large register-based study, Paredes et $\mathrm{al}^{27}$ evaluated the effects of pre-existing mental illness on postoperative outcomes. In that study, patients with severe mental disorders, including schizophrenia, were more likely than controls to have perioperative complications (odds ratio 1.71) and extended durations of hospital admission (odds ratio 2.34), and these differences in morbidity were particularly notable among patients with severe mental illnesses after CABG. ${ }^{27}$ Hauck et $\mathrm{al}^{17}$ investigated the impact of revascularisation after myocardial infarction on mortality among patients with schizophrenia. They used a retrospective cohort of patients with acute myocardial infarction in Ontario between 2008 and 2015, and identified schizophrenia in 1145 patients ( $1.1 \%$ of the total cohort). ${ }^{17}$ The study focused on different outcomes but mainly explored the efficacy of revascularisation therapy for patients with schizophrenia; however, they reported a slightly higher all-cause mortality for patients with schizophrenia after coronary revascularization treatment (CABG and percutaneous coronary intervention combined) compared with the control group (hazard ratio 1.38) in the 1-year follow-up period after acute myocardial infarction. ${ }^{17}$ Our results on mortality among patients with SCZ after CABG were even more favourable: we did not find significant differences in mortality between the groups before 4 years of follow-up. Importantly, our results of poorer outcome in patients with SCZ in longer followup should not be interpreted in the way that would exclude these vulnerable patients from CABG.

Causes for poorer cardiovascular outcomes in SCZ are likely multiple. Patients with schizophrenia smoke considerably more often than the general population, ${ }^{28,29}$ and those who smoke have a 12-fold increased risk of cardiac-related death relative to nonsmokers. ${ }^{30}$ Patients with schizophrenia are also more likely to be obese and have a greater risk of metabolic syndrome. ${ }^{31}$ Visceral obesity in particular is linked to an increased risk of adverse metabolic effects, and individuals with schizophrenia are prone to greater visceral adiposity compared with the general population. ${ }^{32}$ Other unhealthy nutritional and exercise habits might also contribute to the difference between the groups.

Use of evidence-based cardiovascular medication is firmly shown to improve outcomes in CAD ${ }^{18}$ Patients with schizophrenia are at least as adherent to cardiovascular medication as the general population, ${ }^{33}$ yet they are generally less likely to obtain treatment for their somatic conditions. ${ }^{34}$ We found the statins were used significantly less frequently after CABG by patients with SCZ. Furthermore, when statins were used, lower, less effective dosages were more common in patients with SCZ. Of note, the proportion of high-dose statin users was relatively poor $(22 \%)$ in the nonSCZ group as well. However, somewhat surprisingly, the use of renin-angiotensin system inhibitors, beta-blockers, anticoagulants and adenosine diphosphate inhibitors did not differ between study groups. Because effective statin therapy significantly reduces all-cause and cardiovascular mortality after $\mathrm{CABG},{ }^{35,36}$ our results call for more intensive statin therapy after CABG, especially in patients with SCZ.

Antipsychotic medications are the first-line treatment for SCZ. ${ }^{37,38}$ There is strong evidence that the long-term use of antipsychotic medication lowers mortality in patients with SCZ. A recent meta-analysis of studies evaluating antipsychotic medication and mortality of patients with SCZ showed a pooled risk ratio of 0.57 for all-cause mortality with patients using any antipsychotic medication. ${ }^{39}$ We hypothesized that antipsychotic medication consumption would lead to lower mortality after CABG, but found no association of pre-CABG antipsychotic use with outcomes after CABG. However, antipsychotic medications are known for their adverse cardiometabolic effects. ${ }^{40-43}$ It is possible that these metabolic adverse effects, at least in part, balanced the overall adverse effects of weak adherence to antipsychotic medication in this study.

Evidence for polypharmacy in maintenance treatment of SCZ is sparce, but sometimes antipsychotic medications alone are inadequate to address all of the symptoms that occur in individuals with SCZ. ${ }^{44}$ Benzodiazepine medication has been associated with an elevated risk of cardiovascular disease admissions and higher overall mortality in patients with schizophrenia. ${ }^{38}$ However, we did not find significant differences in mortality or MACE for patients that used benzodiazepines before CABG. Psychiatric hospital admission implies severe symptoms in SCZ, and therefore is widely used as marker of unstable mental state in schizophrenia research. ${ }^{1,45}$ Psychiatric hospital admission among patients with SCZ is associated with younger age; prior hospital admission; and comorbidities such as substance misuse, depression, anxiety and personality disorders. ${ }^{46}$ Annual hospital admission rates range from approximately 23 to $27 \%$ in recent literature. ${ }^{46,47}$ In our 
study, the annual psychiatric hospital admission rate was only $7 \%$. Older patient age might have contributed to the low hospital admission rate, but it is also likely that patient selection for CABG favours those with fewer psychiatric symptoms and better overall psychosocial functioning. It is also probable that individuals with more severe psychotic symptoms and comorbidities might avoid invasive treatments or might simply have higher early-age, all-cause mortality. In our data, pre-CABG psychiatric hospital treatment was not associated with mortality or MACE, although the small sample size is likely to yield insufficient statistical power to profoundly evaluate this association. Nevertheless, our results indicate that the need for psychiatric hospital admission or poorer adherence to antipsychotic medications should not be used as categorical grounds for denial of CABG. Further studies are needed on potential influence of CABG to psychiatric symptoms and well-being of patients with SCZ.

This register-based study has limitations. Our study design is retrospective and thus limits the potential to draw definitive conclusions. We used previously validated nationwide registries mandated by law in Finland. ${ }^{48}$ Validity of SCZ diagnosis from these registers has been shown to be reliable. ${ }^{49}$ However, it is still possible that the register data contain sources of bias. All diagnoses in the register were determined by treating clinicians, and there is a possibility of diagnostic or coding errors. Residual confounding by undetermined variables is possible. The prevalence of patients with SCZ diagnoses (ICD-10 groups F20, F21 and F25) in our cohort was only $0.4 \%$, which is somewhat in line with a recent estimate of overall global prevalence of schizophrenia $(0.3 \%),{ }^{50}$ but is considerably lower than a previously reported prevalence of $1.3 \%$ in Finland (for ICD-10 groups F20, F21 and F25 combined). ${ }^{51}$ To improve the accuracy of a SCZ diagnosis, we did exclude diagnoses that might represent nonchronic and potentially aetiologically mixed groups of illnesses, such as ICD-10 code F29 (unspecified psychosis not due to a substance or known physiological condition). We did not have access to laboratory, smoking or income data, which limited the conclusions about the effectors of poorer outcomes. Medication purchases were used as a proxy for medication use, but we did not have direct information of actual use of purchased medications. Our results of CABG-treated patients may not be directly applicable to patients with CAD treated with percutaneous coronary intervention. Also, our sample of patients with SCZ was of limited size, which contributed to the limited statistical power for evaluation of weaker associations.

In conclusion, this study demonstrated that patients with CAD and SCZ are at a higher risk of death and adverse cardiovascular events during long-term follow-up after CABG. Neither psychiatric medication use nor psychiatric hospital admission before CABG were associated with long-term outcomes. Efforts should be targeted to optimise effective secondary prevention of CAD in patients with SCZ after CABG. Patients with chronic psychotic disorders constitute a unique and especially vulnerable subgroup among patients with $\mathrm{CAD}$, and they could benefit from regular cardiovascular out-patient appointments with specialised protocols that encourage them toward healthy lifestyle choices and improved adherence to cardiovascular medication.

Mika Kallio, MD, Department of Psychiatry, University of Turku and Turku University Hospital, Finland; Jyrki Korkeila, MD, PhD, Department of Psychiatry, University of Turku and Turku University Hospital, Finland; and Department of Psychiatry, Hospital District of Satakunta, Finland; Markus Malmberg, MD, PhD, Heart Center, Turku University Hospital and University of Turku, Finland; Jarmo Gunn, MD, PhD, Heart Center, Turku Hospital and University of Turku, Finland; Jarmo Gunn, MD, PhD, Heart Center,
University Hospital and University of Turku, Finland; Päivi Rautava, MD, PhD, Department of Public Health, University of Turku, Finland; and Turku Clinical Research Centre, Turku University Hospital, Finland; Päivi Korhonen, MD, PhD, Turku Clinical Research Centre, Turku University Hospital, Finland; and Department of General Practice, University of Turku and Turku University Hospital, Finland: Ville Kytö (D. MD, PhD, Heart Center, Turku University Hospital and University of Turku, Finland; Research Center of
Applied and Preventive Cardiovascular Medicine, University of Turku, Finland; Center for Population Health Research, Turku University Hospital and University of Turku, Finland; Administrative Center, Hospital District of Southwest Finland, Finland; and Department of Public Health, Faculty of Medicine, University of Helsinki, Finland

Correspondence: Ville Kytö. Email: ville.kyto@utu.fi

First received 1 Nov 2021, final revision 20 Dec 2021, accepted 10 Jan 2022

\section{Supplementary material}

Supplementary material is available online at https://doi.org/10.1192/bjo.2022.10.

\section{Data availability}

The data that support the findings of this study are available on request from the national data authority Findata (wWw. findata.fi), for those fulfilling the requirements. The data are not publicly available because of legal restrictions

\section{Acknowledgements}

None.

\section{Author contributions}

M.K. and V.K. drafted the article. M.K., J.K., M.M., P.K. and V.K. formulated the research question and designed the study. V.K. and P.R. were responsible for data acquisition and study funding. V.K. conducted data analysis and supervised the study. All authors revised the manuscript for V.K. conducted data
intellectual content.

\section{Funding}

This study was supported by grant funding from the Finnish Cultural Foundation, the Finnish Foundation of Cardiovascular Research, the Paulo Foundation and the Finnish Governmental VTR.

Declaration of interest

None.

\section{References}

1 Tiihonen J, Lönnqvist J, Wahlbeck K, Klaukka T, Niskanen L, Tanskanen A, et al. 11-year follow-up of mortality in patients with schizophrenia: a populationbased cohort study (FIN11 study). Lancet 2009; 374: 620-7.

2 Lomholt LH, Andersen DV, Sejrsgaard-Jacobsen C, Øzdemir CM, Graff C, Schjerning $\mathrm{O}$, et al. Mortality rate trends in patients diagnosed with schizophrenia or bipolar disorder: a nationwide study with 20 years of follow-up. Int J Bipolar Disord 2019; 7: 6.

3 Hayes JF, Marston L, Walters K, King MB, Osborn DPJ. Mortality gap for people with bipolar disorder and schizophrenia: UK-based cohort study 2000-2014. Br J Psychiatry 2017; 211: 175-81.

4 Taipale $\mathrm{H}$, Mittendorfer-Rutz E, Alexanderson K, Majak M, Mehtälä J, Hoti F, et al. Antipsychotics and mortality in a nationwide cohort of 29,823 patients with schizophrenia. Schizophr Res 2018; 197: 274-80.

5 Westman J, Eriksson SV, Gissler M, Hällgren J, Prieto ML, Bobo WV, et al. Increased cardiovascular mortality in people with schizophrenia: a 24-year national register study. Epidemiol Psychiatr Sci 2018; 27: 519-27.

6 Correll CU, Solmi M, Veronese N, Bortolato B, Rosson S, Santonastaso P, et al. Prevalence, incidence and mortality from cardiovascular disease in patients with pooled and specific severe mental illness: a large-scale meta-analysis of $3,211,768$ patients and 113,383,368 controls. World Psychiatry 2017; 16: $163-80$.

7 Howell S, Yarovova E, Khwanda A, Rosen SD. Cardiovascular effects of psychotic illnesses and antipsychotic therapy. Heart 2019; 105: 1852-9.

8 De Hert M, Dekker JM, Wood D, Kahl KG, Holt RI, Möller HJ. Cardiovascular disease and diabetes in people with severe mental illness position statement from the European Psychiatric Association (EPA), supported by the European Association for the Study of Diabetes (EASD) and the European Society of Cardiology (ESC). Eur Psychiatry 2009; 24: 412-24.

9 Davidson M. Risk of cardiovascular disease and sudden death in schizophrenia. J Clin Psychiatry 2002; 63(Suppl 9): 5-11.

10 Mohamed MO, Rashid M, Farooq S, Siddiqui N, Parwani P, Shiers D, et al. Acute myocardial infarction in severe mental illness: prevalence, clinical outcomes, and process of care in U.S. hospitalizations. Can J Cardiol 2019; 35: 821-30. 
11 Bodén R, Molin E, Jernberg T, Kieler H, Lindahl B, Sundström J. Higher mortality after myocardial infarction in patients with severe mental illness: a nationwide cohort study. J Intern Med 2015; 277: 727-36.

12 Kugathasan $\mathrm{P}$, Laursen TM, Grøntved S, Jensen SE, Aagaard J, Nielsen RE. Increased long-term mortality after myocardial infarction in patients with schizophrenia. Schizophr Res 2018; 199: 103-8.

13 Mitchell AJ, Lawrence D. Revascularisation and mortality rates following acute coronary syndromes in people with severe mental illness: Comparative metaanalysis. Br J Psychiatry 2011; 198: 434-41.

14 Wu SI, Chen SC, Juang JJM, Fang CK, Liu SI, Sun FJ, et al. Diagnostic procedures, revascularization, and inpatient mortality after acute myocardial infarction in patients with schizophrenia and bipolar disorder. Psychosom Med 2013; 75 52-9.

15 Shao $M$, Tian $H$, Wang L, Jiang D, Feng J, Zhou C. Mortality risk following acute coronary syndrome among patients with schizophrenia: a meta-analysis. Prog Neuropsychopharmacol Biol Psychiatry 2020; 96: 109737.

16 Shao M, Zhuo C, Gao X, Chen C, Xu XY, Tian H, et al. Reduced rate of revascularization in schizophrenic patients with acute myocardial infarction: a systematic review and meta-analysis. Prog Neuropsychopharmacol Biol Psychiatry 2020; 99: 109870.

17 Hauck TS, Liu N, Wijeysundera HC, Kurdyak P. Mortality and revascularization among myocardial infarction patients with schizophrenia: a population-based cohort study. Can J Psychiatry 2020; 65: 454-62.

18 Knuuti J, Winjs W, Saraste A, Capodanno D, Barbato E, Funck-Brentano C, et al. 2019 ESC guidelines for the diagnosis and management of chronic coronary syndromes. Eur Heart J 2020; 41: 407-77.

19 Kytö V, Myllykangas ME, Sipilä J, Niiranen TJ, Rautava P, Gunn J. Long-term outcomes of mechanical Vs biologic aortic valve prosthesis in patients older than 70 years. Ann Thorac Surg 2019; 108: 1354-60.

20 Department of Health and Welfare National Coding Service. ICD-10 Classification list. Elisa, 2019 (https://koodistopalvelu.kanta.fi/codeserver/pages/classification-list-page.xhtml?clearUserCachedLists=true).

21 Kytö V, Ahtela E, Sipilä J, Rautava P, Gunn J. Mechanical versus biological valve prosthesis for surgical aortic valve replacement in patients with infective endocarditis. Interact Cardiovasc Thorac Surg 2019; 29: 386-92.

22 Malmberg M, Gunn J, Sipilä J, Pikkarainen E, Rautava P, Kytö V. Comparison of long-term outcomes of patients having surgical aortic valve replacement with versus without simultaneous coronary artery bypass grafting. Am J Cardiol 2020; 125: 964-9.

23 Olfson M, Gerhard T, Huang C, Crystal S, Stroup TS. Premature mortality among adults with schizophrenia in the United States. JAMA Psychiatry 2015; 72: 1172-81.

24 Hjorthøj C, Stürup AE, McGrath JJ, Nordentoft M. Years of potential life lost and life expectancy in schizophrenia: a systematic review and meta-analysis. Lancet Psychiatry 2017; 4: 295-301.

25 Protty MB, Lacey A, Smith D, Hannoodee S, Freeman P. Increased morbidity, mortality and length of in-hospital stay for patients with acute coronary syndrome with pre-morbid psychiatric diagnoses. Int J Cardiol 2017; 236: 5-8.

26 Attar R, Valentin JB, Freeman P, Andell P, Aagaard J, Jensen SE. The effect of schizophrenia on major adverse cardiac events, length of hospital stay, and prevalence of somatic comorbidities following acute coronary syndrome. Eur Hear J Qual Care Clin Outcomes 2019; 5: 121-6.

27 Paredes AZ, Hyer JM, Diaz A, Tsilimigras DI, Pawlik TM. The impact of mental illness on postoperative outcomes among Medicare beneficiaries: a missed opportunity to help surgical patients? Ann Surg 2020; 272(3): 419-25.

28 Lohr JB, Flynn K. Smoking and schizophrenia. Schizophr Res 1992; 8: 93-102.

29 Krieger I, Tzur Bitan D, Comaneshter D, Cohen A, Feingold D. Increased risk of smoking-related illnesses in schizophrenia patients: a nationwide cohort study. Schizophr Res 2019; 212: 121-5.

30 Kelly DL, McMahon RP, Wehring HJ, Liu F, Mackowick KM, Boggs DL, et al Cigarette smoking and mortality risk in people with schizophrenia. Schizophr Bull 2011; 37: 832-8.

31 Must A, Spadano J, Coakley EH, Field AE, Colditz G, Dietz WH. The disease burden associated with overweight and obesity. J Am Med AssoC 1999; 282: 1523-9.

32 Thakore JH, Mann JN, Vlahos I, Martin A, Reznek R. Increased visceral fat distribution in drug-naive and drug-free patients with schizophrenia. Int J Obes 2002; 26: $137-41$
33 Owen-Smith A, Stewart C, Green C, Ahmedani BK, Waitzfelder BE, Rossom R, et al. Adherence to common cardiovascular medications in patients with schizophrenia vs. patients without psychiatric illness. Gen Hosp Psychiatry 2016; 38: 9-14.

34 Laursen TM, Munk-Olsen T, Gasse C. Chronic somatic comorbidity and excess mortality due to natural causes in persons with schizophrenia or bipolar affective disorder. PLoS One 2011; 6: e24597.

35 Papathanasiou A, Toumpoulis IK, Milionis HJ, Kalantzi K, Katsouras CS, Goudevenos J. Statin therapy is associated with reduced total and cardiovascular mortality after coronary artery bypass grafting surgery. Coron Artery Dis 2008; 19: 619-25.

36 Alkhalil M. Effects of intensive lipid-lowering therapy on mortality after coronary bypass surgery: a meta-analysis of 7 randomised trials. Atherosclerosis 2020; 293: 75-8

37 Lally J, MacCabe JH. Antipsychotic medication in schizophrenia: a review. Br Med Bull 2015; 114: 169-79.

38 Stroup TS, Gerhard T, Crystal S, Huang C, Tan C, Wall ME, et al. Comparative effectiveness of adjunctive psychotropic medications in patients with schizophrenia. JAMA Psychiatry 2019; 76: 508

39 Vermeulen J, Van Rooijen G, Doedens P, Numminen E, van Tricht M, de Haan L. Antipsychotic medication and long-term mortality risk in patients with schizophrenia: a systematic review and meta-analysis. Psychol Med 2017; 47: 2217-28.

40 Pillinger T, McCutcheon RA, Vano L, Mizuno Y, Arumuham A, Hindley G, et al. Comparative effects of 18 antipsychotics on metabolic function in patients with schizophrenia, predictors of metabolic dysregulation, and association with psychopathology: a systematic review and network meta-analysis. Lancet Psychiatry 2020; 7: 64-77.

41 Huhn M, Nikolakopoulou A, Schneider-Thoma J, Krause M, Samara M, Peter N, et al. Comparative efficacy and tolerability of 32 oral antipsychotics for the acute treatment of adults with multi-episode schizophrenia: a systematic review and network meta-analysis. Lancet 2019; 394: 939-51.

42 Rummel-Kluge $\mathrm{C}$, Komossa $\mathrm{K}$, Schwarz $\mathrm{S}$, Hunger $\mathrm{H}$, Scmid F, Lobos CA, et al. Head-to-head comparisons of metabolic side effects of second generation antipsychotics in the treatment of schizophrenia: a systematic review and metaanalysis. Schizophr Res 2010; 123: 225-33.

43 Komossa K, Rummel-Kluge C, Hunger H, Schmid F, Schwarz S, Duggan L, et al. Olanzapine versus other atypical antipsychotics for schizophrenia. Cochrane Database Syst Rev 2010; 3: CD006654.

44 Baandrup L. Polypharmacy in schizophrenia. Basic Clin Pharmacol Toxicol 2020; 126: 183-92.

45 Kishimoto T, Hagi K, Nitta M, Leucht S, Olfson M, Kane JM, et al. Effectiveness of long-acting injectable vs oral antipsychotics in patients with schizophrenia: a meta-analysis of prospective and retrospective cohort studies. Schizophr Bull 2018; 44: 603-19.

46 Yan T, Greene M, Chang E, Broder MS, Touya M, Munday J, et al. Hospitalization risk factors in antipsychotic-treated schizophrenia, bipolar I disorder or major depressive disorder. J Comp Eff Res 2019; 8: 217-27.

47 Lang K, Federico V, Muser E, Menzin J, Menzin J. Rates and predictors of antipsychotic non-adherence and hospitalization in Medicaid and commerciallyinsured patients with schizophrenia. J Med Econ 2013; 16: 997-1006.

48 Sund R. Quality of the Finnish Hospital Discharge Register: a systematic review. Scand J Public Health 2012; 40: 505-15.

49 Pihlajamaa J, Suvisaari J, Henriksson M, Heilä H, Karjalainen E, Koskela J, et al. The validity of schizophrenia diagnosis in the Finnish Hospital Discharge Register: findings from a 10-year birth cohort sample. Nord J Psychiatry 2008; 62: 198-203.

50 Charlson FJ, Ferrari AJ, Santomauro DF, Diminic S, Stockings E, Scott JG, et al. Global epidemiology and burden of schizophrenia: findings from the Global Burden of Disease Study 2016. Schizophr Bull 2018; 44: 1195-203.

51 Perälä J, Suvisaari J, Saarni SI, Kuoppasalmi K, Isometsä E, Pirkola S, et al. Lifetime prevalence of psychotic and bipolar I disorders in a general population. Arch Gen Psychiatry 2007; 64: 19-28. 